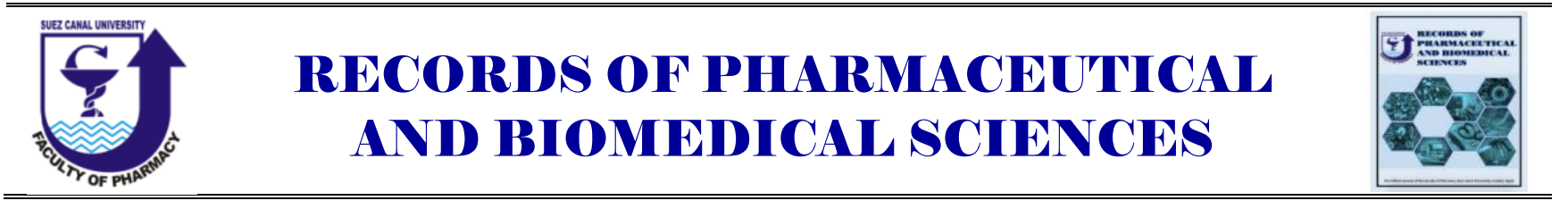

\title{
Nano-formulations for Different Treatment Modalities to Overcome Multi Drug Resistance in Hepatocellular Carcinoma
}

\author{
Hend E. Abo Mansour ${ }^{a,}$, Maha M. El-Batsh ${ }^{b}$, Nadia S. Badawy ${ }^{c}$,Eman T. Mehanna ${ }^{d}$, Noha M. Mesbah ${ }^{d}$, \\ Dina M. Abo-Elmatty \\ ${ }^{a}$ Department of Biochemistry, Faculty of Pharmacy, Menoufia University, Egypt, ${ }^{b}$ Department of Clinical \\ Pharmacology, Faculty of Medicine, Menoufia University, Egypt, ${ }^{c}$ Department of Histology and Cell \\ Biology, Faculty of Medicine, Menoufia University, Egypt, ${ }^{d}$ Department of Biochemistry, Faculty of \\ Pharmacy, Suez Canal University, Ismailia, Egypt
}

Received on: 27. 03. 2020

Revised on: 17. 04. 2020

Accepted on: 23. 04. 2020

Correspondence Author:

Tel: +201098505161

E-mail address:

hend elsaid@phrm.menofia.edu.eg

\begin{abstract}
Hepatocellular carcinoma (HCC) is one of the most common malignancies and it is the fourth cause of cancer-related mortality. The treatment of HCC with traditional chemotherapeutic agents such as doxorubicin has been limited due to poor efficacy, acquired resistance of the tumors and systemic toxicity. Verapamil as a P-gp inhibitor has demonstrated anti-cancer activities both in vitro and in vivo. Natural products and plant-derived compounds, such as ginger extract, have been investigated as safe and effective anti-cancer agents. Combination therapy offers preferable prognosis with less side effects. For example, the combination of chemotherapeutics with verapamil or ginger extract has been shown to exert beneficial therapeutic effects, overcome multidrug resistance and reduce side effects through modulation of different signaling pathways in tumor cells. Nano-sized formulations are designed to increase accumulation of drugs at the tumor environment and to improve solubility/bioavailability, promote retention, specificity, efficacy and safety. This review summarizes how to overcome cardiotoxicity and multidrug resistance evolved by doxorubicin in HCC treatment.
\end{abstract}

Key words: Doxorubicin, Ginger extract, Hepatocellular carcinoma, Nano-sized formulations, Verapamil.

\section{Epidemiology of HCC}

More than $80 \%$ of HCC cases are in countries with limited medical and social care resources, especially in Eastern Asia and sub-Saharan Africa.
HCC accounts for more than $90 \%$ of primary liver tumors and is responsible for 700 thousands deaths per year (Yang et al. 2019). The incidence of HCC varies according to exposure to environmental and infectious risk factors, availability of healthcare 
resources, and ability to detect earlier HCC stage (Geh et al. 2019). Higher distribution occurs in sub-Saharan Africa and Eastern Asia with incidence rates of more than 20 per 100 thousands individuals (Johnson 2019). Intermediate incidence rates of 10-20 per 100 thousands individuals are in Mediterranean countries such as Greece, Italy and Spain. While the low incidence rates of less than 5 per 100 thousands individuals occur in north and south America (Yu et al. 2015). Liver cancer is highly increased among men in Middle East countries, particularly Egypt and Saudi Arabia. Recent reports demonstrate that the incidence of HCC has increased sharply in Egypt through the last 5-10 years (Sharafi and Alavian 2020)

\section{Risk Factors for HCC}

Stimulatory effect of androgen and the protective effect of estrogen induce higher incidence of HCC in males than females (Tian et al. 2015). Hepatitis B virus (HBV) and hepatitis $\mathrm{C}$ virus (HCV) infections promote cirrhosis, and account for $80-90 \%$ of patients with HCC (Petruzziello 2018). Cirrhosis is one of the primary risk factors for developing HCC. It develops after long periods of chronic liver disease where liver cells are destroyed and fibrous tissues are formed. Cirrhosis has a remarkable effect on liver regenerative capacity and is often an essential part of the mortality associated with HCC (Zoheiry et al. 2015).

Alcohol abuse leads to cirrhosis and HCC development. Alcohol is mainly metabolized in the liver to acetaldehyde and acetic acid by alcohol dehydrogenase and aldehyde dehydrogenase, respectively.
Free radical release and lipid peroxidation generally contribute to alcoholic liver disease (Farzaei et al. 2018). Obesity usually results from fat accumulation in the body and elevates the risk for HCC. Lipid accumulation in obesity increases interleukin-6 (IL-6) and tumor necrosis factor- $\alpha$ (TNF- $\alpha$ ) expression and creates a status of oxidative stress, which can induce DNA damage and genomic instability (Chitapanarux and Phornphutkul 2015).

Aflatoxin is produced by the fungal species Aspergillus flavus, Aspergillus parasiticus and Aspergillus nominus. Aflatoxin induces HCC through proto-oncogen activation and tumor suppressor gene p53 inactivation, DNA synthesis interruption, various chromosomal aberrations, and chromosomal strand breaks in human cells (Maurya and Trigun 2016). Smoking has a carcinogenic potential through metabolism of its ingredients in the liver. The association between tobacco and HCC depends on many factors as sex, genetics, and underlying history of viral hepatitis (Boccia et al. 2015).

Sex hormones usually modulate liver function. Prolonged use of oral contraceptives and androgenic steroids can induce benign and malignant carcinoma (Buettner and Thimme 2019). Hereditary hemochromatosis is the condition in which iron is overloaded and induces liver cancer. HCC develops in $10 \%$ of patients with hereditary hemochromatosis. The tumorigenic effect of excessive iron is mediated through reactive oxygen species (ROS) that damage DNA, lipids and 
proteins resulting in liver cell necrosis (Muto et al. 2019).

\section{Molecular Pathogenesis of HCC}

\subsection{Proliferation pathways}

Like several malignancies, HCC development can be considered as an imbalance between the programmed cell death process (apoptosis) and uncontrolled proliferation (Lei et al. 2015). The cell cycle is a series of steps that a cell must progress through in order to proliferate. It is tightly controlled by many important checkpoints. These checkpoints assess DNA damage and extracellular growth signals and ensure that damaged DNA is not passed on to prospective daughter cells (Mirzayans et al. 2016).

Tumor development and progression is a stepwise process involving initiation and promotion. Initiation occurs spontaneously or by an endogenous ROS or exogenous mutagen (tobacco smoke). Initiating agents cause DNA damage, activate proto-oncogenes (Ras) and inactivate tumor suppressor genes (p53) (Lehmann et al. 2016). However, initiation will not give rise to a cancer on its own; a promoter is needed. A promoter is a compound that can promote tumor growth when subsequently applied to an initiating factor (Lehmann et al. 2016).

\subsection{Apoptotic pathways}

Apoptosis (programmed cell death) is proposed to be death of a cell in any pathological format, when mediated by an intracellular program (Tiwari and Khan 2016). Two core pathways exist to induce apoptosis, the extrinsic death receptor pathway and intrinsic mitochondrial pathway (Figure 1). The extrinsic pathway is triggered by binding of fatty acid synthase (Fas) with its extracellular ligand (Fas-L). Upon exposure to death stimuli, the Fas/Fas-L composite recruits death domaincontaining protein and pro-caspase- 8 , forming the death-inducing signaling complex. Consequently, pro-caspase- 8 and pro-caspase-3 are activated, which proceed to trigger cell death (Huang et al. 2016).

The intrinsic pathway also leads to apoptosis but under the control of mitochondrial pro-enzymes (Figure 1). Outer mitochondrial membranes become permeable to internal cytochrome $\mathrm{c}$ and release it into the cytosol. Cytochrome $\mathrm{c}$ recruits apoptotic peptidase activating factor 1 (Apaf-1) and pro-caspase-9 to compose the apoptosome, which downstream activates caspase 9/3 signaling cascade, resulting in apoptosis. Several evidences have shown that abnormal expression of some key regulatory factors may lead to cancer, indicating the essential relationship between apoptosis and cancer (Tiwari and Khan 2016).

\subsection{Oxidative stress}

Oxidative stress is determined by the rates of both ROS production and scavenging. Cells are normally able to protect themselves against ROS damage through the use of specific enzymatic (peroxidase, dismutase and catalase) or nonenzymatic (A, C, and $\mathrm{E}$ vitamins and glutathione) ROS-reducing mechanisms (Khurana et al. 2018). Oxidative stress could be induced via chronic hepatic inflammation regardless of etiology. Acute liver injury and hepatic inflammation induce 


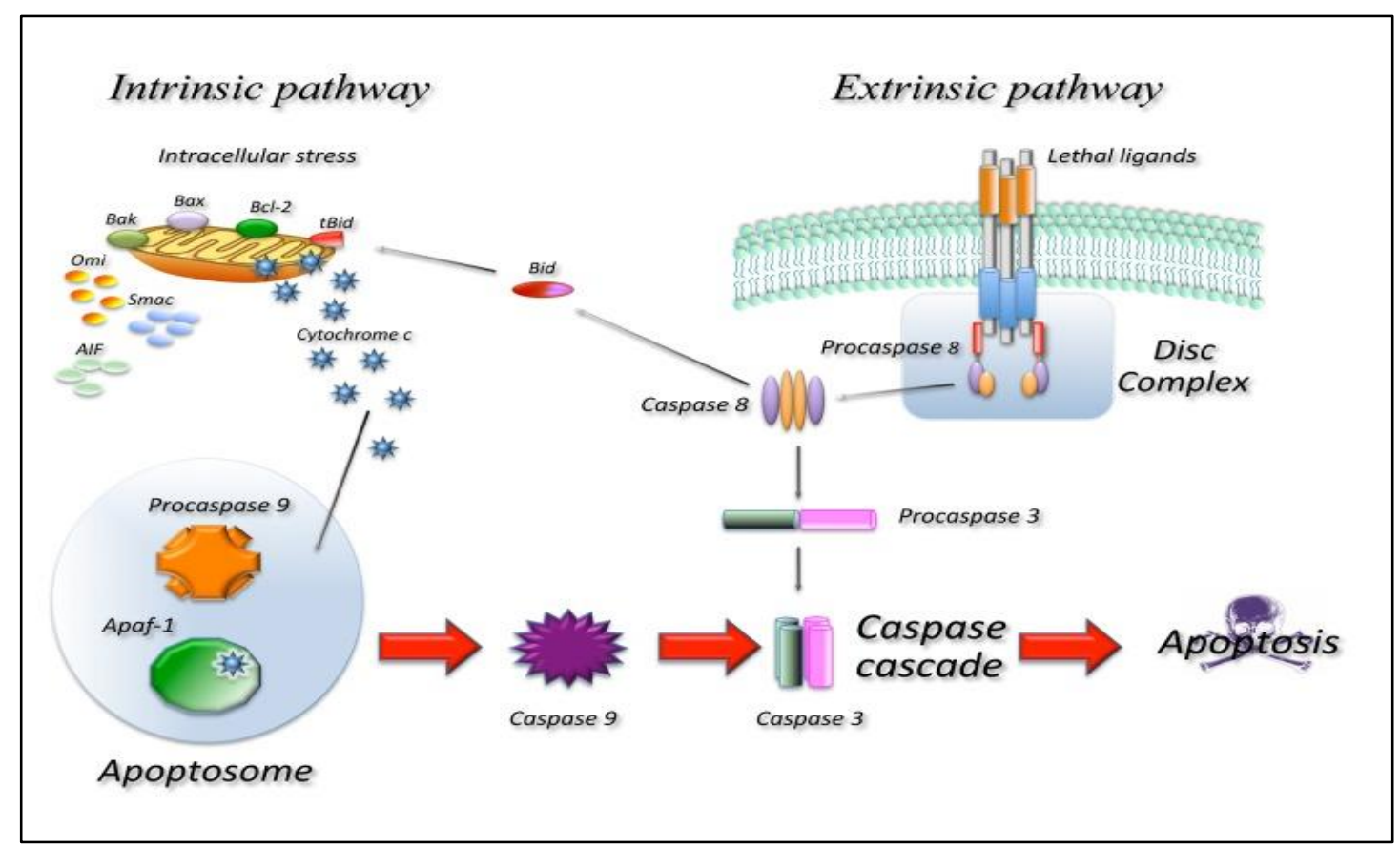

Figure (1): The main molecular pathways leading to apoptosis. Apaf-1: apoptotic protease activating factor (Favaloro et al. 2012).

ROS via neutrophils and Kupffer cell activation (Ramos-Tovar et al. 2018). The ROS in mitochondria are by-products of fatty acid beta oxidation and can be generated via electron leakage from mitochondrial electron transport resulting in the oxidative modifications in proteins, lipids and DNA and activation of oncogenic pathways (Siegenthaler and Sevier 2019).

\section{Multidrug resistance in $\mathrm{HCC}$}

Multidrug resistance (MDR) is the protection of cancer cells against numerous drugs differing in chemical structure and cellular mechanisms of action. MDR is one of the major causes of chemotherapy failures in treatment of human tumors (Robey et al. 2018). Drug resistance may arise from acquired and/or intrinsic factors. Acquired drug resistance is influenced by environmental or genetic factors that enhance development of drug-resistant cancer cells or induce mutations of essential enzymes in the metabolic pathways (Mansoori et al. 2017).

On the other hand, intrinsic resistance can be attributed to drug breakdown or modified drug transport across the cell membrane (Mansoori et al. 2017). ATP-dependent transporters are implicated in transporting nutrients and other molecules across the membrane. ATP binding cassette (ABC) family has three members, including P-glycoprotein (P$\mathrm{gp})$, multi-drug resistance-associated protein 1 (MRP1) and breast cancer resistance protein (BCRP/ABCG2) (Robey et al. 2018). Multidrug resistance protein 1 (MDR1) was identified as the first mammalian $\mathrm{ABC}$ multidrug transporter when the Chinese hamster ovary cells displayed pleiotropic cross-resistance to a wide range of amphiphilic drugs (Juliano and Ling 1976). 
This transporter was named P-glycoprotein (P-gp) and is also known as ABCB1 (Binkhathlan and Lavasanifar 2013).

The expression pattern of P-gp indicates that its main function is the protection of the body from xenobiotic and toxic substances by excreting them into bile, urine, feces and avoiding their entry into fetus and brain. P-gp is expressed in the liver, kidneys, intestine, brain and testes (Kim and Chen 2018). Besides this physiological function, P-gp inhibits the delivery of many drugs to their targets, such as anti-cancer drugs, immunosuppressants, cardiac glycosides, statins, anti-hypertensive, calcium channel blockers and antibiotics. It prevents cellular internalization of chemotherapeutic agents and makes the chemotherapy ineffective in many cases. Therfore, this protein is one of the main barriers in cancer treatment by chemotherapy (Marcelletti et al. 2019).

\section{Therapeutic Strategies for HCC}

\section{Chemotherapy (Doxorubicin as an example)}

$\mathrm{HCC}$ is advanced disease that is primarily managed by surgery, radiotherapy, hormonal therapy or chemotherapy. Doxorubicin (DOX) is related to broad spectrum antibiotics, called anthracyclines (Gnapareddy et al. 2015). DOX has been used to treat several types of tumors such as bone sarcomas, acute leukaemia, breast carcinoma, neuroblastoma, lymphoma, thyroid carcinoma, lung carcinoma, liver and ovarian carcinoma (Matvienko et al. 2019). Oxidative stress, the disruption of topoisomerase II-mediated DNA repair and intercalation into DNA, are the mechanisms by which DOX exerts its anticancer activities (Mobaraki et al. 2017) .
DOX is a hydrophobic molecule that passes through cellular membranes independently of specific transporters. However, cells can fail to accumulate it through active drug efflux via ATPdependent efflux transporters. Overexpression of $\mathrm{P}$ gp in hepatocytes can prevent cell internalization of DOX and can induce resistance in $\mathrm{HCC}$ ( $\mathrm{Li}$ et al. 2015). Cardiotoxicity is related to free radical formation caused by DOX metabolism (Giampieri et al. 2016). DOX reduction by NADH dehydrogenase forms a semiquinone radical that can react with molecular oxygen to form the superoxide radicals. DOX can form ferric ionDOX complexes, which further catalyze the conversion of hydrogen peroxide to several ROS species (Fenton's reaction). Cardiac tissues are much more sensitive to the oxidative stress caused by DOX because of their great dependence on oxidative substrate metabolism. ROS subsequently cause cell death through apoptotic pathways activation (Henninger and Fritz 2017; Reis-Mendes et al. 2015) (Figure 2).

The biochemical markers of DOX-induced cardiotoxicity include lactate dehydrogenase $(\mathrm{LDH})$, creatine kinase isoenzyme MB (CK-MB), and troponin I(QuanJun et al. 2017). The multidrug resistance and systemic toxicity of DOX can limit its clinical application (Kocahan et al. 2017). Therefore, there is a great need to use new strategies to improve DOX efficacy and reduce its cardiotoxicity. These strategies could include using P-gp inhibitors, complementary natural therapy and new drug delivery systems (Cote et al. 2015; González et al. 2017). 


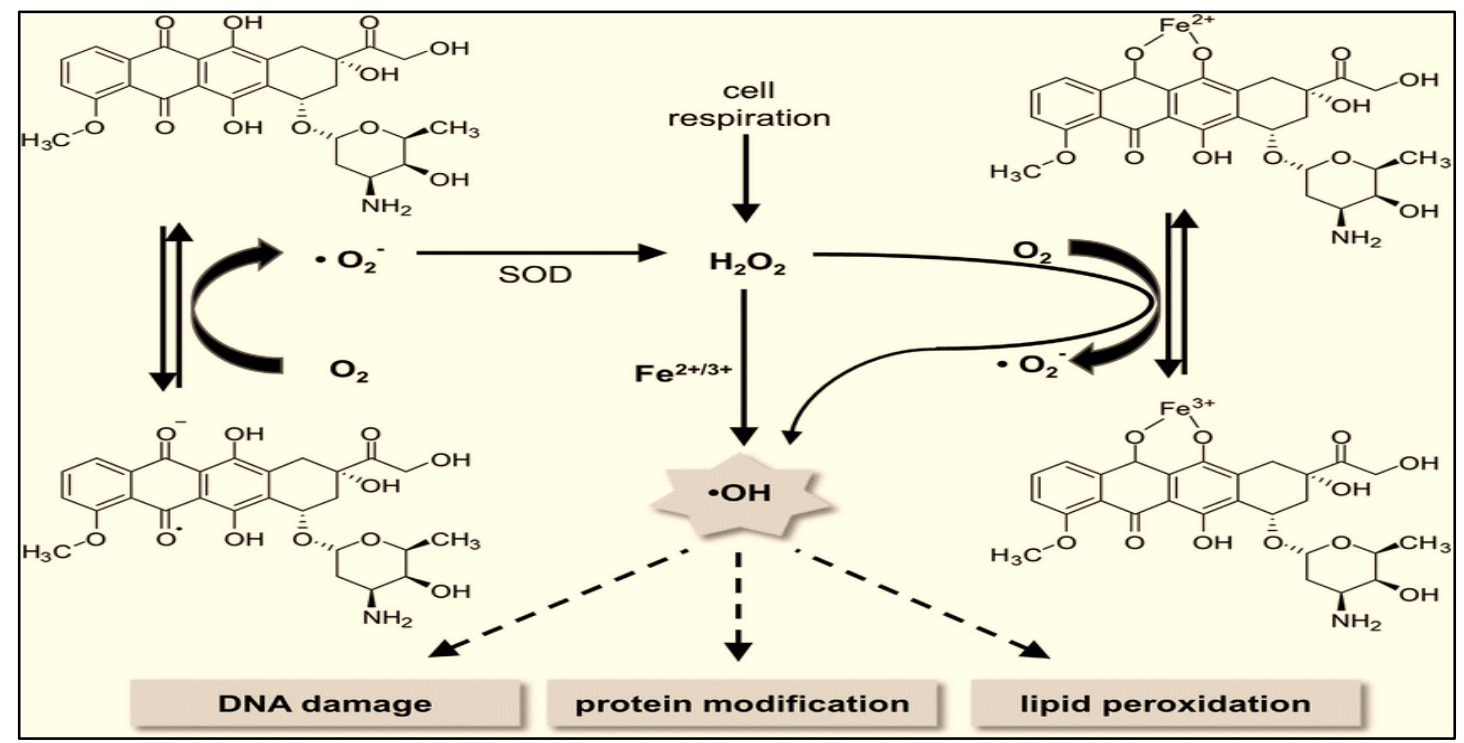

Figure (2): Model of doxorubicin-mediated generation of ROS by redox cycling and Fenton's reaction (Henninger and Fritz 2017).

\section{P-gp inhibitors}

Efflux pump inhibition is mainly done to improve the delivery of therapeutic agents. P-gp inhibitors are classified into three generations based on their specificity, affinity, and toxicity. First generation inhibitors are pharmacologically active substances which are clinically used for specific treatments but have the ability to inhibit P-gp. The use of first generation is limited owing to their high serum concentrations (at the doses that are required to inhibit P-gp) and potential toxicity (Yu et al. 2016). Second generation lacks the pharmacological activities and possesses higher P-gp affinity which includes cyclosporin A and dexverapamil. However, these inhibitors lead to pharmacokinetic alterations through inhibition of the metabolizing CYPA4 enzyme and other ABC transporters. The third generation is under clinical development, aiming to inhibit P-gp with higher specificity and lower toxicity such as tariquidar (Hoosain et al. 2015).Complementary therapy is widely used as an aid for management of several diseases such as
Verapamil (VRP), a calcium channel blocker, treats cardiac arrhythmias through inhibition of calcium ion flux (Zhao et al. 2016). Several studies have described the role of VRP in growth inhibition of tumor cells including lung and bone cancer cells and meningioma (Zhang et al. 2009; Karsy et al. 2016; Fernandes et al. 2016). VRP can be used as an adjuvant in multi-drug chemotherapy through inhibition of P-gp and calmodulin activity in cancer cells; thus, it can diminish drug resistance (Chen et al. 2014; Baek and Cho 2015). However, use of VRP is restricted because very high doses are needed to inhibit P-gp, and this causes cardiac side effects (Abdallah et al. 2015). Fortunately, these limitations can be overcome by using nano-carriers (Patel et al. 2013).

\section{Complementary natural therapy}

The use of complementary and alternative medicine (CAM) has been growing alongside conventional medical treatments. 
cancer (River et al. 2018). Ginger (Zingiber officinale), has been widely used as a spice in foods and a medication for digestive disorders. Pungent components; such as gingerols, shogaols, paradols and zingerone; have antioxidant, antidiabetic, antiinflammatory and anticancer activities (Mao et al. 2019). Ginger and its phenolic constituents have been shown to inhibit proliferation and induce apoptosis in several tumor types in vitro and in vivo (Prasad and Tyagi 2015; Rahman et al. 2011; ElAshmawy et al. 2018).
Ginger constituents mediate their anti-cancer activity via signaling pathways and molecules (Figure 3). Ginger extract (GE) up-regulates expression of antioxidant enzymes such as glutathione peroxidase (GPx), catalase (CAT) and superoxide dismutase (SOD), and increases their activity (Jeena et al. 2013). GE decreases expression of the TNF- $\alpha$, vascular endothelial growth factor (VEGF) and nuclear factor-kappa B (NF-kB) (Tsuboi et al. 2014). Furthermore, GE activates adenosine monophosphate protein kinase, down-regulates cyclin D1 and increases tumor suppressor gene p53 (El-Ashmawy et al. 2018).

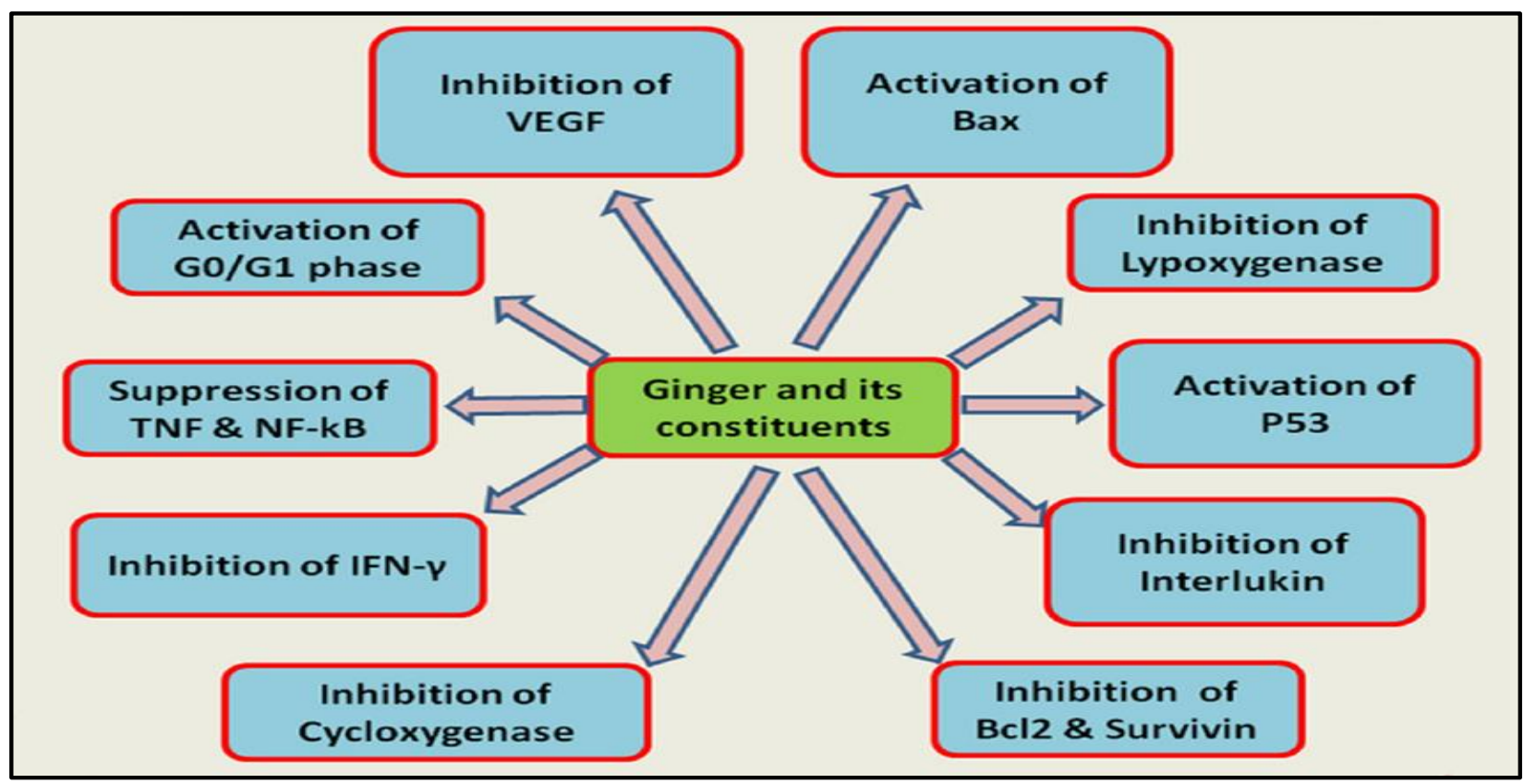

Figure (3): Anti-cancer mechanisms of ginger and its constituents. Bcl-2: B-cell lymphoma 2, Bax: Bcl-2associated x protein, IFN- $\gamma$ : interferon- $\gamma, \mathrm{NF}-\mathrm{\kappa B}$ : nuclear factor-kappa B, TNF: tumor necrosis factor, VEGF: vascular endothelial growth factor (Rahmani et al. 2014).

\section{Nanotechnology}

Nanoparticle (NP)-based targeted drug delivery is a rapidly progressing field of cancer research aiming to solve many limitations of conventional drug delivery systems. Conventional chemotherapy has less aqueous solubility, lacks selectivity for the cancer cells and exhibits MDR (Chen et al. 2018). Therefore, nanoparticles loaded with DOX and VRP can be a useful strategy in cancer therapy (Zhang et al. 2017; Zheng et al. 2018). NP formulations with chemotherapeutics and natural products have higher therapeutic index 
due to enhanced delivery to tumor tissues, thereby promoting permeability, retention, pharmacokinetic profiles, and reducing side effects. NPs reduce permeability across healthy capillaries, which in turn decrease bioavailability and toxicity to normal tissue (Caster et al. 2015). Several types of nanocarriers are used in tumor-targeted delivery including micelles, liposomes, carbon nanotubes and polymeric nanoparticles (Sebastian 2017) Bioabsorbable polymers undergo transformations in the biological environment through cellular activity. The bioabsorbable materials that are commonly used in cancer therapy are polyesters, polyanhydrides, polysaccharides (e.g., chitosan, dextran, hyaluronic acid), and proteins (e.g., albumin, gelatin) (Fonseca et al. 2015).

Chitosan is a polysaccharide of biological origin. The marine biopolymer chitin, transforms into chitosan by partial deacetylation under strong alkaline conditions. Chitosan is composed of $(\mathrm{N}$ acetyl D-glucosamine) and (D-glucosamine) units, and has been used in health care materials, such as wound healing preparations and nasal absorption enhancers of peptide drugs (Babu and Ramesh 2017). Chitosan exhibits high biocompatibility, biodegradability, and attractive properties for the development of active and safe drug delivery tools. Chitosan is cationic in nature and its solubility in water is weak but it is soluble in acidic solutions (Saikia et al. 2015). The cationic charge of chitosan has been used for ionic gelation methods using materials with anionic charge for nanoparticle preparation. This cationic nature has been harnessed for electrostatic interaction with nucleic acids, and chitosan has been used as a gene delivery carrier for cancer treatment (Kumar et al. 2015).
Nano-formulations of combined treatment with DOX and VRP or DOX and GE can overcome multi drug resistance, enhance DOX anti-cancer activity and reduce its cardiotoxicity. Therefore, this therapeutic strategy has great promise in clinical application for HCC management.

\section{Conflict of interest}

All authors have no conflict of interest

\section{References}

Abdallah, H. M., A. M. Al-Abd, R. S. El-Dine, and A. M. El-Halawany. 2015. P-glycoprotein inhibitors of natural origin as potential tumor chemo-sensitizers: A review. Journal of Advanced Research 6 (1):45-62.

Babu, A., and R. Ramesh. 2017. Multifaceted Applications of Chitosan in Cancer Drug Delivery and Therapy. Marine drugs 15 (4):96.

Baek, J. S., and C. W. Cho. 2015. Controlled release and reversal of multidrug resistance by coencapsulation of paclitaxel and verapamil in solid lipid nanoparticles. Int J Pharm 478 (2):617-624.

Binkhathlan, Z., and A. Lavasanifar. 2013. Pglycoprotein inhibition as a therapeutic approach for overcoming multidrug resistance in cancer: current status and future perspectives. Curr Cancer Drug Targets 13 (3):326-346.

Boccia, S., L. Miele, and N. Panic. 2015. The effect of CYP, GST, and SULT polymorphisms and their interaction with smoking on the risk of hepatocellular carcinoma. 2015:179867.

Buettner, N., and R. Thimme. 2019. Sexual dimorphism in hepatitis $\mathrm{B}$ and $\mathrm{C}$ and hepatocellular carcinoma. Semin Immunopathol 41 (2):203-211.

Caster, J. M., M. Sethi, S. Kowalczyk, E. Wang, X. Tian, S. Nabeel Hyder, K. T. Wagner, Y. A. Zhang, 
C. Kapadia, K. Man Au, and A. Z. Wang. 2015. Nanoparticle delivery of chemosensitizers improve chemotherapy efficacy without incurring additional toxicity. Nanoscale 7 (6):2805-2811.

Chen, S., R. Li, X. Li, and J. Xie. 2018. Electrospinning: An enabling nanotechnology platform for drug delivery and regenerative medicine. Adv Drug Deliv Rev 132:188-213.

Chen, Y., X. L. Zheng, D. L. Fang, Y. Yang, J. K. Zhang, H. L. Li, B. Xu, Y. Lei, K. Ren, and X. R. Song. 2014. Dual agent loaded PLGA nanoparticles enhanced antitumor activity in a multidrug-resistant breast tumor eenograft model. Int J Mol Sci 15 (2):2761-2772.

Chitapanarux, T., and K. Phornphutkul. 2015. Risk Factors for the Development of Hepatocellular Carcinoma in Thailand. J Clin Transl Hepatol 3 (3):182-188.

Cote, B., L. J. Carlson, D. A. Rao, and A. W. G. Alani. 2015. Combinatorial resveratrol and quercetin polymeric micelles mitigate doxorubicin induced cardiotoxicity in vitro and in vivo. $J$ Control Release 213:128-133.

El-Ashmawy, N. E., N. F. Khedr, H. A. ElBahrawy, and H. E. Abo Mansour. 2018. Ginger extract adjuvant to doxorubicin in mammary carcinoma: study of some molecular mechanisms. Eur J Nutr 57 (3):981-989.

Farzaei, M. H., M. Zobeiri, F. Parvizi, and F. F. ElSenduny. 2018. Curcumin in Liver Diseases: A Systematic Review of the Cellular Mechanisms of Oxidative Stress and Clinical Perspective. 10 (7).

Favaloro, B., N. Allocati, V. Graziano, C. Di Ilio, and V. De Laurenzi. 2012. Role of apoptosis in disease. Aging 4 (5):330-349.
Fernandes, G., A. Barone, and R. Dziak. 2016. Effects of verapamil on bone cancer cells. Journal of Cell-Biology-\&-Cell-Metabolism 3:13.

Fonseca, A. C., A. C. Serra, and J. F. J. Coelho. 2015. Bioabsorbable polymers in cancer therapy: latest developments. EPMA Journal 6 (1):22.

Geh, D., F. A. Rana, and H. L. Reeves. 2019. Weighing the benefits of hepatocellular carcinoma surveillance against potential harms. J Hepatocell Carcinoma 6:23-30.

Giampieri, F., J. M. Alvarez-Suarez, M. Gasparrini, T. Y. Forbes-Hernandez, S. Afrin, S. Bompadre, C. Rubini, A. Zizzi, P. Astolfi, C. Santos-Buelga, A. M. Gonzalez-Paramas, J. L. Quiles, B. Mezzetti, and M. Battino. 2016. Strawberry consumption alleviates doxorubicin-induced toxicity by suppressing oxidative stress. Food Chem Toxicol 94:128-137.

Gnapareddy, B., S. R. Dugasani, T. Ha, B. Paulson, T. Hwang, T. Kim, J. H. Kim, K. Oh, and S. H. Park. 2015. Chemical and Physical Characteristics of Doxorubicin Hydrochloride Drug-Doped Salmon DNA Thin Films. Sci Rep 5:12722.

González, M. L., D. M. A. Vera, J. Laiolo, M. B. Joray, M. Maccioni, S. M. Palacios, G. Molina, P. A. Lanza, S. Gancedo, V. Rumjanek, and M. C. Carpinella. 2017. Mechanism Underlying the Reversal of Drug Resistance in P-GlycoproteinExpressing Leukemia Cells by Pinoresinol and the Study of a Derivative. Frontiers in pharmacology 8:205-205.

Henninger, C., and G. Fritz. 2017. Statins in anthracycline-induced cardiotoxicity: Rac and Rho, and the heartbreakers. Cell Death Dis 8 (1):e2564. Hoosain, F. G., Y. E. Choonara, L. K. Tomar, P. 
Kumar, C. Tyagi, L. C. du Toit, and V. Pillay. 2015. Bypassing P-glycoprotein drug efflux mechanisms: possible applications in pharmacoresistant schizophrenia therapy. Biomed Res Int 2015.

Huang, Y., M. Yang, H. Hu, X. Zhao, L. Bao, D. Huang, L. Song, and Y. Li. 2016. Mitochondrial GRIM-19 as a potential therapeutic target for STAT3-dependent carcinogenesis of gastric cancer. Oncotarget 7 (27):41404-41420.

Jeena, K., V. B. Liju, and R. Kuttan. 2013. Antioxidant, anti-inflammatory and antinociceptive activities of essential oil from ginger. Indian $J$ Physiol Pharmacol 57 (1):51-62.

Johnson, P. 2019. The Epidemiology of Hepatocellular Carcinoma. In Liver Cancers: Springer, 3-11.

Juliano, R. L., and V. Ling. 1976. A surface glycoprotein modulating drug permeability in Chinese hamster ovary cell mutants. Biochim Biophys Acta 455 (1):152-162.

Karsy, M., N. Hoang, T. Barth, L. Burt, W. Dunson, D. L. Gillespie, and R. L. Jensen. 2016. Combined Hydroxyurea and Verapamil in the Clinical Treatment of Refractory Meningioma: Human and Orthotopic Xenograft Studies. World Neurosurg 86:210-219.

Khurana, R. K., A. Jain, A. Jain, T. Sharma, B. Singh, and P. Kesharwani. 2018. Administration of antioxidants in cancer: debate of the decade. Drug Discov Today 23 (4):763-770.

Kim, Y., and J. Chen. 2018. Molecular structure of human P-glycoprotein in the ATP-bound, outwardfacing conformation. Science 359 (6378):915-919. Kocahan, S., Z. Dogan, E. Erdemli, and E. Taskin. 2017. Protective Effect of Quercetin Against
Oxidative Stress-induced Toxicity Associated With Doxorubicin and Cyclophosphamide in Rat Kidney and Liver Tissue. Iran J Kidney Dis 11 (2):124131.

Kumar, S., P. Garg, S. Pandey, M. Kumari, S. Hoon, K.-J. Jang, R. Kapavarapu, P.-H. Choung, A. J. Sobral, and J. H. Chung. 2015. Enhanced chitosan-DNA interaction by 2-acrylamido-2methylpropane coupling for an efficient transfection in cancer cells. Journal of Materials Chemistry B 3 (17):3465-3475.

Lehmann, C., T. Friess, F. Birzele, A. Kiialainen, and M. Dangl. 2016. Superior anti-tumor activity of the MDM2 antagonist idasanutlin and the Bcl-2 inhibitor venetoclax in p53 wild-type acute myeloid leukemia models. J Hematol Oncol 9 (1):50.

Lei, B., W. Chai, Z. Wang, and R. Liu. 2015. Highly expressed UNC119 promotes hepatocellular carcinoma cell proliferation through Wnt/betacatenin signaling and predicts a poor prognosis. $A m$ J Cancer Res 5 (10):3123-3134.

Li, N., P. Zhang, C. Huang, Y. Song, S. Garg, and Y. Luan. 2015. Co-delivery of doxorubicin hydrochloride and verapamil hydrochloride by $\mathrm{pH}$ sensitive polymersomes for the reversal of multidrug resistance. RSC Advances 5 (95):7798677995.

Mansoori, B., A. Mohammadi, S. Davudian, S. Shirjang, and B. Baradaran. 2017. The Different Mechanisms of Cancer Drug Resistance: A Brief Review. Adv Pharm Bull 7 (3):339-348.

Mao, Q. Q., X. Y. Xu, S. Y. Cao, R. Y. Gan, H. Corke, T. Beta, and H. B. Li. 2019. Bioactive Compounds and Bioactivities of Ginger (Zingiber 
officinale Roscoe). Foods 8 (6).

Marcelletti, J. F., B. I. Sikic, L. D. Cripe, and E. Paietta. 2019. Evidence of a role for functional heterogeneity in multidrug resistance transporters in clinical trials of P-glycoprotein modulation in acute myeloid leukemia. Cytometry B Clin Cytom 96 (1):57-66.

Matvienko, T., V. Sokolova, S. Prylutska, Y. Harahuts, N. Kutsevol, V. Kostjukov, and M. Evstigneev. 2019. In vitro study of the anticancer activity of various doxorubicin-containing dispersions. 9 (1):57-63.

Maurya, B. K., and S. K. Trigun. 2016. Fisetin Modulates Antioxidant Enzymes and Inflammatory Factors to Inhibit Aflatoxin-B1 Induced Hepatocellular Carcinoma in Rats. Oxid Med Cell Longev 2016:1972793.

Mirzayans, R., B. Andrais, P. Kumar, and D. Murray. 2016. The Growing Complexity of Cancer Cell Response to DNA-Damaging Agents: Caspase 3 Mediates Cell Death or Survival? Int J Mol Sci 17 (5).

Mobaraki, M., A. Faraji, M. Zare, P. Dolati, M. Ataei, and H. D. Manshadi. 2017. Molecular mechanisms of cardiotoxicity: a review on major side-effect of doxorubicin. Indian journal of pharmaceutical sciences 79 (3):335-344.

Muto, Y., T. Moroishi, K. Ichihara, M. Nishiyama, H. Shimizu, H. Eguchi, K. Moriya, K. Koike, K. Mimori, M. Mori, Y. Katayama, and K. I. Nakayama. 2019. Disruption of FBXL5-mediated cellular iron homeostasis promotes liver carcinogenesis. The Journal of experimental medicine 216 (4):950-965.

Patel, N. R., B. S. Pattni, A. H. Abouzeid, and V. P.
Torchilin. 2013. Nanopreparations to overcome multidrug resistance in cancer. Adv Drug Deliv Rev 65 (13-14):1748-1762.

Petruzziello, A. 2018. Epidemiology of Hepatitis B Virus (HBV) and Hepatitis C Virus (HCV) Related Hepatocellular Carcinoma. The open virology journal 12:26-32.

Prasad, S., and A. K. Tyagi. 2015. Ginger and its constituents: role in prevention and treatment of gastrointestinal cancer. Gastroenterol Res Pract 2015:142979.

QuanJun, Y., Y. GenJin, W. LiLi, H. YongLong, H. Yan, L. Jie, H. JinLu, L. Jin, G. Run, and G. Cheng. 2017. Protective effects of dexrazoxane against doxorubicin-induced cardiotoxicity: a metabolomic study. PLoS One 12 (1).

Rahman, S., F. Salehin, and A. Iqbal. 2011. In vitro antioxidant and anticancer activity of young Zingiber officinale against human breast carcinoma cell lines. BMC Complement Altern Med 11:76.

Rahmani, A. H., F. M. Shabrmi, and S. M. Aly. 2014. Active ingredients of ginger as potential candidates in the prevention and treatment of diseases via modulation of biological activities. Int J Physiol Pathophysiol Pharmacol 6 (2):125-136.

Ramos-Tovar, E., E. Hernandez-Aquino, S. Casas-

Grajales, L. D. Buendia-Montano, and S. GalindoGomez. 2018. Stevia Prevents Acute and Chronic Liver Injury Induced by Carbon Tetrachloride by Blocking Oxidative Stress through Nrf2 Upregulation. 2018:3823426.

Reis-Mendes, A. F., E. Sousa, M. de Lourdes Bastos, and V. M. Costa. 2015. The Role of the Metabolism of Anticancer Drugs in Their InducedCardiotoxicity. Curr Drug Metab 17 (1):75-90. 
River, J., H. McKenzie, D. Levy, N. Pavlakis, M. Back, and B. Oh. 2018. Convergent priorities and tensions: a qualitative study of the integration of complementary and alternative therapies with conventional cancer treatment. Support Care Cancer 26 (6):1791-1797.

Robey, R. W., K. M. Pluchino, and M. D. Hall. 2018. Revisiting the role of $\mathrm{ABC}$ transporters in multidrug-resistant cancer. 18 (7):452-464.

Saikia, C., P. Gogoi, and T. Maji. 2015. Chitosan: a promising biopolymer in drug delivery applications. J. Mol. Genet. Med. S 4 (006).

Sebastian, R. 2017. Nanomedicine-the Future of cancer treatment: A review. J. Cancer Prev. Curr. Res 8 (1):00-265.

Sharafi, H., and S. M. Alavian. 2020. The Rising Threat of Hepatocellular Carcinoma in the Middle East and North Africa Region: Results From Global Burden of Disease Study 2017. Clinical liver disease 14 (6):219-223.

Siegenthaler, K. D., and C. S. Sevier. 2019. Working Together: Redox Signaling between the Endoplasmic Reticulum and Mitochondria. 32 (3):342-344.

Tian, Y. E., X. U. Xie, Y. Lin, G. Tan, and W. U. Zhong. 2015. Androgen receptor in hepatocarcinogenesis: Recent developments and perspectives. Oncol Lett 9 (5):1983-1988.

Tiwari, P., and M. J. Khan. 2016. Molecular and Computational Studies on Apoptotic Pathway Regulator, Bcl-2 Gene from Breast Cancer Cell Line MCF-7. Indian journal of pharmaceutical sciences 78 (1):87-93.

Tsuboi, K., Y. Matsuo, T. Shamoto, T. Shibata, S. Koide, M. Morimoto, S. Guha, B. Sung, B. B.
Aggarwal, H. Takahashi, and H. Takeyama. 2014. Zerumbone inhibits tumor angiogenesis via NFkappaB in gastric cancer. Oncol Rep 31 (1):57-64. Yang, J. D., P. Hainaut, and G. J. Gores. 2019. A global view of hepatocellular carcinoma: trends, risk, prevention and management. 16 (10):589604.

Yu, G., X. Chen, S. Chen, W. Ye, K. Hou, and M. Liang. 2015. Arsenic trioxide reduces chemoresistance to 5-fluorouracil and cisplatin in $\mathrm{HBx}$ HepG2 cells via complex mechanisms. Cancer Cell Int 15:116.

Yu, J., P. Zhou, J. Asenso, X. D. Yang, C. Wang, and W. Wei. 2016. Advances in plant-based inhibitors of P-glycoprotein. J Enzyme Inhib Med Chem 31 (6):867-881.

Zhang, C., F. Lv, L. Zhou, X. Li, X. X. Wu, and R. M. Hoffman. 2009. Effect of verapamil on the expression of EGFR and NM23 in A549 human lung cancer cells. Anticancer Res 29 (1):27-32.

Zhang, M., E. Liu, Y. Cui, and Y. Huang. 2017. Nanotechnology-based combination therapy for overcoming multidrug-resistant cancer. Cancer biology \& medicine 14 (3):212-227.

Zhao, L., Y. Zhao, B. Schwarz, J. Mysliwietz, R. Hartig, P. Camaj, Q. Bao, K. W. Jauch, M. Guba, J. W. Ellwart, P. J. Nelson, and C. J. Bruns. 2016. Verapamil inhibits tumor progression of chemotherapy-resistant pancreatic cancer side population cells. Int J Oncol 49 (1):99-110.

Zheng, W., M. Li, Y. Lin, and X. Zhan. 2018. Encapsulation of verapamil and doxorubicin by MPEG-PLA to reverse drug resistance in ovarian cancer. Biomed Pharmacother 108:565-573. Zoheiry, M. M., S. A. Hasan, E. El-Ahwany, F. M. 
Nagy, H. A. Taleb, M. Nosseir, M. Magdy, S. Meshaal, M. D. El-Talkawy, and I. Raafat. 2015. Serum Markers of Epithelial Mesenchymal Transition as Predictors of HCV-induced Liver Fibrosis, Cirrhosis and Hepatocellular Carcinoma. Electron Physician 7 (8):1626-1637. 